\section{IN BRIEF}

\section{$\Rightarrow$ SYSTEMIC LUPUS ERYTHEMATOSUS}

\section{Belimumab slows organ damage progression}

To assess the efficacy of long-term treatment with belimumab, a post-hoc longitudinal study has used propensity score matching to compare patients from the BLISS long-term extension study who received belimumab (and standard of care) with patients from the Toronto Lupus Cohort who received standard of care only. Belimumab treatment resulted in less organ damage progression (as assessed by change in SLICC/ ACR Damage Index (SDI) score from baseline to 5 years), as well as a reduced likelihood of progression to a higher SDI score over any given year, compared with standard of care.

ORIGINAL ARTICLE Urowitz, M. B. et al. Organ damage in patients treated with belimumab versus standard of care: a propensity score-matched comparative analysis. Ann. Rheum. Dis. https://doi.org/10.1136/annrheumdis-2018-214043 (2019)

\section{$\Rightarrow$ SYSTEMIC SCLEROSIS}

\section{Lung biomarkers in SSc: prognostic or diagnostic?}

In the largest study of its kind to date, involving 427 patients from two cohorts, the performance and optimal positioning of candidate serum biomarkers of interstitial lung disease (ILD) in systemic sclerosis (SSc) was assessed. SP-D, KL-6 and CCL18 performed best as biomarkers for ILD diagnosis, lung fibrosis severity and ILD progression, respectively. A matrix model that combined baseline concentrations of SP-D and anti-topoisomerase I antibody positivity could accurately stratify patients on the basis of their risk of fibrosis, whereas a model that incorporated CCL18 levels could stratify patients with SSc-ILD on the basis of their risk of ILD progression. ORIGINAL ARTICLE Elhai, M. et al. Performance of candidate serum biomarkers for systemic sclerosis-interstitial lung disease. Arthritis Rheumatol. https://doi.org/10.1002/ art.40815 (2019)

\section{$\Rightarrow$ RHEUMATOID ARTHRITIS}

\section{Assisted reproduction less effective in RA}

Women with rheumatoid arthritis (RA) are less likely to have a live birth following assisted reproduction technology (ART) treatment than women without RA (adjusted OR 0.78; $95 \% \mathrm{Cl} 0.65-0.92)$ according to a nationwide study that analysed data from the Danish ART registry (1,149 and 198,941 embryo transfers in women with and without RA, respectively). Women with RA had a reduced chance of a biochemical pregnancy and a clinical pregnancy after embryo transfer, suggesting a reduced likelihood of embryo implantation.

ORIGINAL ARTICLE Nørgård, B. M. et al. Decreased chance of a live born child in women with rheumatoid arthritis after assisted reproduction treatment: a nationwide cohort study. Ann. Rheum. Dis. https://doi.org/10.1136/annrheumdis-2018-214619 (2019)

\section{$\Rightarrow$ OSTEOARTHRITIS}

\section{OA genetic risk loci more than doubled}

In a new osteoarthritis (OA) genome-wide meta-analysis that used data from the UK Biobank and the Arthritis Research UK Osteoarthritis Genetics (arcOGEN) consortium, which included $\sim 17.5$ million single-nucleotide variants in $>450,000$ individuals of European descent, 64 genome-wide-significant regions have been identified. This study adds 52 new loci to the list of 34 already-known disease-associated loci in OA, including 24 for OA at any site, 15 for hip OA, 7 for knee OA and 6 for hip and/or knee OA. Ten of the putative effector genes are targets of approved therapeutics or therapeutics in clinical trials.

ORIGINAL ARTICLE Tachmazidou, I. et al. Identification of new therapeutic targets for osteoarthritis through genome-wide analyses of UK Biobank data. Nat. Genet. 51, 230-236 (2019)

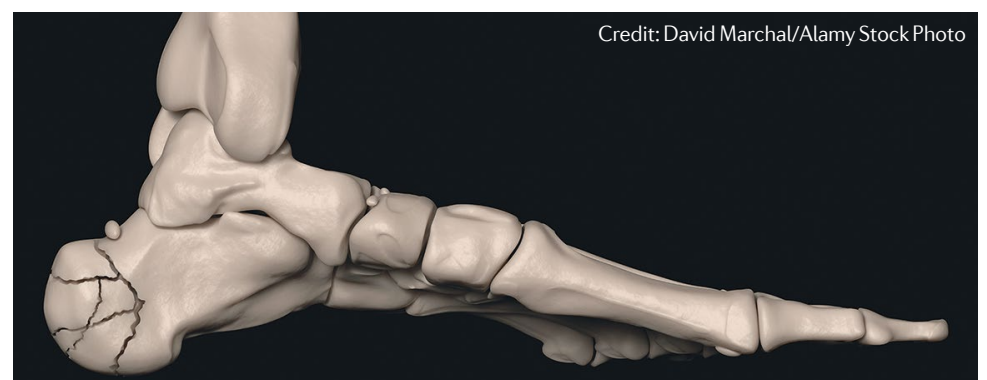

OSTEOPOROSIS

\title{
GWAS cracks fracture risk
}

Bone mineral density (BMD) is a heritable risk factor for osteoporosis and bone fracture, both of which constitute common and serious health concerns in the elderly. A new study published in Nature Genetics uses a genome-wide association study (GWAS) for estimated BMD (eBMD) to provide an atlas of associated genetic loci and lays the foundation for further analyses of a set of targetable genes for prevention of fracture.

The researchers utilized the UK Biobank cohort, which provides strong statistical power for association testing thanks to the large sample size; eBMD in this cohort was measured by quantitative ultrasound of the heel bone.

The first GWAS in $>400,000$ individuals identified 515 loci (301 of which were novel) associated with eBMD. Although the individual effects on eBMD are small, together these loci explain $\sim 20 \%$ of the variation in bone density in the general population.

In a second GWAS, the researchers analysed fracture risk itself and identified 13 loci in UK Biobank participants, classified into cases $(n=53,184)$ and controls $(n=373,611)$ using hospital records. All 13 loci were replicated in a cohort of research participants from the personal genetics company 23andMe $(n=731,819)$ and all 13 loci were also associated with eBMD with genome-wide significance in the initial GWAS.

To shorten the list to potential causal genes, the authors applied a number of statistical methods to obtain a set of fine-mapped singlenucleotide polymorphisms (SNPs). These SNPs were overlapped with ATAC-sequencing data on chromatin accessibility in an osteosarcoma cell line and ENCODE tracks of DNase I hypersensitivity sites in human primary osteoblasts. Then, in conjunction with knowledge of genomic features of genes with known function in bone biology, a set of target genes was generated for functional analysis.

A high-throughput screen of knockout mice from the Origins of Bone and Cartilage Disease study showed that loss of any given selected target gene $(n=126)$ more often resulted in an abnormal skeletal phenotype than when a gene from an unselected set of genes $(n=526)$ was knocked out.

Taking the combined evidence from the eBMD GWAS, fracture-risk GWAS, statistical fine-mapping and mouse skeletal phenotype into account, DAAM2 (which encodes a protein involved in $\mathrm{Wnt} / \beta$-catenin signalling) was selected as an example target gene for validation.

The researchers showed that CRISPR-Cas9-mediated disruption of $D A A M 2$ reduced mineralization in human osteoblast cells in vitro. Furthermore, Daam2-knockout mice had high cortical porosity and reduced bone strength, validating this gene as a potential target for prevention or treatment of osteoporosis.

"We expect that this work will be used to highlight further targets for drug development," says Brent Richards, one of the corresponding authors "Furthermore, this work might help us identify people with genetically increased risk of osteoporosis".

Michelle Trenkmann, Associate Editor Nature Communications

ORIGINAL ARTICLE Morris, J. A. et al. An atlas of genetic influences on osteoporosis in humans and mice. Nat. Genet. https://doi.org/10.1038/ 541588-018-0302-x (2018) 